\title{
ON THE MÜNTZ-SZÁSZ THEOREM FOR $C[0,1]$
}

\author{
ALAN R. SIEGEL ${ }^{1}$
}

\begin{abstract}
The functions $1, t^{\lambda_{1}}, t^{\lambda_{2}}, \cdots$ with complex $\lambda$ 's are shown to be incomplete in $C[0,1]$ under conditions weaker than those proven by Szász, and a special construction due to P. D. Lax where the functions are complete is given.
\end{abstract}

In 1916 Szász proved the following classical result:

THEOREM 1. Suppose $\operatorname{Re} \lambda_{j}>0, j=1,2, \cdots$, and, for the sake of simplicity, the $\lambda$ 's are distinct. Then the functions $1, t^{\lambda_{1}}, t^{\lambda_{2}}, \cdots$ are complete in $C[0,1]$ if

and incomplete if

$$
\sum \frac{\operatorname{Re} \lambda_{j}}{1+\left|\lambda_{j}\right|^{2}}=\infty
$$

$$
\sum \frac{\operatorname{Re} \hat{\lambda}_{j}+1}{1+\left|\lambda_{j}\right|^{2}}<\infty .
$$

When lim inf ${ }_{j \rightarrow \infty} \operatorname{Re} \lambda_{j}>0,(1)$ is the negation of (2) and constitutes a necessary and sufficient condition for completeness. However, under circumstances where neither (1) nor (2) is satisfied, for example if $\lambda_{j}=1 / j+i \sqrt{ } j$ or the $\lambda$ 's are bounded and $\sum \operatorname{Re} \lambda_{j}<\infty$, the completeness question is not answered by Szász's theorem.

For the $L^{2}$ case, it should be noted that there is no such unresolved margin. This is because the $L^{2}$ distance between $t^{\lambda}$ and the linear span of $\left\{t^{\lambda}\right\}_{j=1}^{\infty}$ can be explicitly expressed in terms of a Blaschke product which converges to a nontrivial function if and only if

$$
\sum \frac{\operatorname{Re} \lambda_{j}+\frac{1}{2}}{1+\left|\lambda_{j}\right|^{2}}<\infty
$$

The purpose of this paper is to reduce the gap in Theorem 1 .

TheOREM 2. Suppose $\operatorname{Re} \lambda_{j}>0, j=1,2, \cdots, \lambda_{j} \rightarrow \infty$ as $j \rightarrow \infty$ and the $\lambda$ 's are distinct. If, for some $\alpha<1$,

$$
\sum \frac{\operatorname{Re} \lambda_{j}+\exp \left(-\left|\hat{\lambda}_{j}\right|^{\alpha}\right)}{1+\left|\hat{\lambda}_{j}\right|^{2}}<\infty,
$$

Received by the editors September 1, 1971.

AMS 1970 subject classifications. Primary 41A30, 30A18.

${ }^{1}$ Research supported by the Atomic Energy Commission, contract \#AT(11-1)3077.

(c. American Mathematical Society 1972 
then the linear span of $1, t^{\dot{\lambda}_{1}}, t^{\dot{\prime}_{2}}, \cdots$ is not dense in $C[0,1]$. In fact, $t^{\dot{\lambda}}$ cannot be uniformly approximated by elements of the span unless $\lambda$ equals one of the $\lambda_{j}$ 's or 0 .

Proof. We shall construct a bounded linear functional $l$ on $C[0,1]$, see (14), such that $l\left(t^{\dot{\lambda}}\right)=0$ only when $\lambda=0, \lambda_{1}, \lambda_{2}, \cdots$. First, a few preliminary constructions. Set

$$
e^{-s}=t, \quad F(s)=f\left(e^{-s}\right)
$$

for $f \in C[0,1]$. Then $F(s) \in C[0, \infty]$ and has a Laplace transform

$$
\hat{F}(w)=\int_{0}^{\infty} e^{x s} F(s) d s
$$

for $\operatorname{Re} w<0$. Clearly $\hat{F}$ satisfies

$$
|\hat{F}(w)| \leqq \frac{\|F\|_{\infty}}{-\operatorname{Re} w} .
$$

Lemma. Choose some $\beta, \alpha<\beta<1$, and, via the Poisson integral, define an analytic function $\psi$ in the right half-plane such that

$$
\operatorname{Re} \psi(i y)=\exp \left(-|y|^{\beta}\right) .
$$

Then $\psi$ has the following properties:

(a) $\psi$ is bounded.

(b) The mapping $z \rightarrow w$ defined by

$$
w=z-\psi(z)
$$

maps the right half-plane $1-1$ onto a region containing $\operatorname{Re} w \geqq 0$.

(c) Denote by $\gamma_{j}$ the point whose image under this map is $\lambda_{j}$ :

$$
\lambda_{j}=\gamma_{j}-\psi\left(\gamma_{j}\right)
$$

Then

$$
\operatorname{Re} \gamma_{j}=O\left(\operatorname{Re} \lambda_{j}+\exp \left(-\left|\lambda_{j}\right|^{\alpha}\right)\right)
$$

Proof later.

It follows from (4) and (11) that the Blaschke product

$$
B(z)=\prod_{j=0}^{\infty} \frac{\gamma_{j}-z}{\bar{\gamma}_{j}+z} \frac{\bar{\gamma}_{j}}{\gamma_{j}}=\prod_{j=0}^{\infty}\left(1-\frac{2 z \operatorname{Re} \gamma}{\left|\gamma_{j}\right|^{2}+\gamma_{j} z}\right)
$$

converges. From (7) and (8), we have

$$
|\hat{F}(i y-\psi(i y))| \leqq \exp \left(|y|^{\beta}\right)\|F\|_{\infty} .
$$


Choosing a $\delta, \alpha<\beta<\delta<1$, we define the linear functional $l(f)$ as follows:

$$
l(f)=\int_{-i \infty}^{i \infty} \hat{F}(z-\psi(z)) \exp \left(-z^{\delta}\right) B(z) d z .
$$

Since $|B(i y)|=1$ and $\left|\exp \left(|y|^{\beta}\right) \exp \left(-(i y)^{\delta}\right)\right|$ is integrable, it follows from (13) that $l$ is bounded. Now set $f=t^{\lambda}$; then $F(s)=e^{-\lambda s}, \hat{F}(w)=1 /(\lambda-w)$, and $\hat{F}(z-\psi(z))=-1 /(z-\psi(z)-\lambda)$ is meromorphic with one pole, $\gamma$, in the right half-plane. In this case, the contour in (14) can be shifted to the right, with the total contribution resulting from the residue at $z=\gamma$ since the integrand vanishes in a dominated way at infinity:

$$
l\left(t^{\hat{\lambda}}\right)=2 \pi i \frac{\exp \left(-\gamma^{\delta}\right) B(\gamma)}{1-\psi^{\prime}(\gamma)} .
$$

Therefore $l\left(t^{\dot{\lambda}}\right)$ vanishes if and only if $B$ vanishes at $\gamma$; this happens only when $\gamma=\gamma_{0}, \gamma_{1}, \cdots$, i.e., when $\lambda=0, \lambda_{1}, \lambda_{2}, \cdots$. This completes the proof except for the lemma.

Setting

$$
\psi(x+i y)=\frac{1}{\pi} \int_{-\infty}^{\infty} \frac{e^{-|t|^{\beta}}}{x+i(y-t)} d t
$$

gives property (8) and the boundedness of $\operatorname{Re} \psi$. But

$$
\begin{aligned}
|\operatorname{Im} \psi(x+i y)| & =\left|\frac{1}{\pi} \int_{-\infty}^{\infty} \frac{e^{-|t|^{\beta}}(t-y)}{x^{2}+(y-t)^{2}} d t\right| \\
& =\left|\frac{1}{\pi} \int_{0}^{\infty} \frac{t\left(e^{-|t+y|^{\beta}}-e^{-|t-y|^{\beta}}\right)}{x^{2}+t^{2}} d t\right| \\
& <\sup _{y} \int_{0}^{1}\left|\frac{e^{-|t+y|^{\beta}}-e^{-|t-y|^{\beta}}}{t}\right| d t+\int_{-\infty}^{\infty} e^{-|t|^{\beta}} d t \\
& <2 \beta \sup _{y} \int_{0}^{1} \frac{e^{-|t-y|^{\beta}}}{|t-y|^{1-\beta}} d t+\int_{-\infty}^{\infty} e^{-|t| \beta} d t<\infty .
\end{aligned}
$$

So $|\psi(z)|<k$ for some $k$, which is property (a). Applying the argument principle to $z-\psi(z)$ in the right half-plane gives (b). Now

$$
\begin{aligned}
\operatorname{Re} \psi(z) & =\frac{1}{\pi} \int_{-\infty}^{\infty} \frac{x e^{-|t|^{\beta}}}{x^{2}+(y-t)^{2}} d t \\
& \leqq \frac{1}{\pi} \int_{-|y / 2|}^{|y / 2|} \frac{e^{-|t|^{\beta}} x}{x^{2}+(y-t)^{2}} d t+\frac{e^{-|y / 2|^{\beta}}}{\pi} \int_{-\infty}^{\infty} \frac{x}{x^{2}+(y-t)^{2}} d t \\
& <\frac{4 x|y|}{\pi\left(x^{2}+y^{2}\right)}+e^{-|y / 2|^{\beta}} \\
\operatorname{Re} & \psi(z)<\operatorname{Re}(z / 2)+\exp \left(-|\operatorname{Im}(z / 2)|^{\beta}\right), \quad|z| \text { large. }
\end{aligned}
$$


But $\gamma_{j}=\hat{\lambda}_{j}+\psi\left(\gamma_{j}\right)$, and hence $\operatorname{Re} \gamma_{j}=\operatorname{Re} \lambda_{j}+\operatorname{Re} \psi\left(\gamma_{j}\right)$. Applying (16) yields

$$
\operatorname{Re} \gamma_{j}=O\left(\operatorname{Re} \lambda_{j}+\exp \left(-\left|\operatorname{Im}\left(\gamma_{j} / 2\right)\right|^{\beta}\right)\right)
$$

Since $\left|\lambda_{j}-\gamma_{j}\right|<k$, it follows that

$$
\exp \left(-\left|\operatorname{Im}\left(\gamma_{j} / 2\right)\right|^{\beta}\right)=O\left(\operatorname{Re} \hat{\lambda}_{j}+\exp \left(-\left|\gamma_{j}\right|^{\alpha}\right)\right), \quad \alpha<\beta .
$$

Combining (17) and (18) gives (11), the final part of the lemma.

Corollary. Suppose $\operatorname{Re} \lambda_{j}>0, j=1,2, \cdots, \lambda_{j} \rightarrow i \gamma$ as $j \rightarrow \infty$ and the $\lambda$ 's are distinct. If, for some $\alpha<1$,

$$
\sum \operatorname{Re} \lambda_{j}+\exp \left(-\left|\frac{1}{\lambda_{j}-i \gamma}\right|^{\alpha}\right)<\infty,
$$

then $1, t^{\lambda_{1}}, t^{\lambda_{2}}, \cdots$ is not complete in $C[0,1]$.

This follows from setting

$$
l(f)=\int_{-i \infty}^{i \infty} \hat{F}\left(i \gamma+\frac{1}{z-\psi(z)}\right) e^{-z^{\delta}} B(z) d z .
$$

CoRollary. Suppose $\lambda_{j, k} \rightarrow i \gamma_{k}$ and $\lambda_{j, 0} \rightarrow \infty$ as $j \rightarrow \infty, k=1,2, \cdots, l$, the $\lambda$ 's are distinct and with positive real parts. If, for some $\alpha<1$,

$$
\sum_{j=1}^{\infty} \operatorname{Re}\left[\frac{\lambda_{j .0}+\exp \left(-\left|\lambda_{j, 0}\right|^{\alpha}\right)}{1+\left|\lambda_{j .0}\right|^{2}}+\sum_{k=1}^{l} \lambda_{j, k}+\exp \left(-\left|\frac{1}{\lambda_{j, k}-i \gamma_{k}}\right|^{\alpha}\right)\right]<\infty,
$$

then the functions $1, t^{\lambda_{j, k}}, j=1,2, \cdots, k=0,1, \cdots, l$, are not complete in $C[0,1]$.

The result is a direct consequence of setting

$$
\begin{aligned}
& \hat{F}\left(w_{0}, w_{1}, \cdots, w_{l}\right)=\int_{0}^{\infty} \int_{0}^{\infty} \cdots \int_{0}^{\infty} \exp \left(w_{0} s_{0}+w_{1} s_{1}+\cdots+w_{l} s_{l}\right) \\
& \times F\left(s_{0}+s_{1}+\cdots+s_{l}\right) d s_{0} d s_{1} \cdots d s_{l}, \\
& l(f)=\int_{-i \infty}^{i \infty} \int_{-i \infty}^{i \infty} \cdots \int_{-i \infty}^{i \infty} \hat{F}\left(z_{0}-\psi\left(z_{0}\right),\right. \\
& \left.i \gamma_{1}+\frac{1}{z_{1}-\psi\left(z_{1}\right)}, \cdots, i \gamma_{l}+\frac{1}{z_{l}-\psi\left(z_{l}\right)}\right) \\
& \times \prod_{k=0}^{l} e^{-z}{ }_{k}^{\delta} B_{k}\left(z_{k}\right) d z_{k} .
\end{aligned}
$$

COROLlaRY. Suppose the $\lambda_{j}$ 's are positive and distinct for $j=1,2, \cdots$. Then $1, t^{\lambda_{1}}, t^{\lambda_{2}}, \cdots$ are complete in $C[0,1]$ if and only if

$$
\sum\left[\hat{\lambda}_{j} /\left(1+\hat{\lambda}_{j}^{2}\right)\right]=\infty \text {. }
$$


This generalization of Müntz's theorem is stated, though not proven, in [2, p. 29]. Its proof may be simplified by letting $\psi \equiv 1$ in the preceding calculations.

For complex $\lambda$ 's, however, Lax has shown by example that (1) is not necessary for completeness.

THEOREM 3 (LAX). Let $l_{j}$ be any sequence of positive numbers going to infinity, and $\alpha_{j}$ any positive sequence tending to zero. Define $\lambda_{j, k}$ by

$$
\lambda_{j, k}=\alpha_{j}+i 2 \pi k / l_{j}, \quad j=1,2, \cdots, k=0, \pm 1, \pm 2, \cdots .
$$

Then $\left\{1, t^{\lambda_{j, k}}\right\}_{j=1}^{\infty} \underset{k=-\infty}{\infty}$ is complete in $C[0,1]$.

If we choose $\alpha_{j}$ and $l_{j}$ so that

$$
\sum \alpha_{j} l_{j}<\infty
$$

then $\sum_{j, k}\left[\operatorname{Re} \lambda_{j, k} /\left(1+\left|\lambda_{j, k}\right|^{2}\right)\right]$ converges since

$$
\sum_{j=1}^{\infty} \sum_{k=-\infty}^{\infty} \frac{\operatorname{Re} \hat{\lambda}_{i, k}}{1+\left|\lambda_{j, k}\right|^{2}}<\sum_{j=1}^{\infty} \alpha_{j}\left(1+2 \int_{0}^{\infty} \frac{d x}{1+\left(x / l_{j}\right)^{2}}\right)=O\left(\sum \alpha_{j} l_{j}\right) .
$$

Unfortunately, this example is somewhat pathological since the accumulation points of $\lambda_{j, k}$ are precisely the imaginary axis.

Proof of THEOREM 3. Let $X$ be the closure in $C_{0}[0, \infty]$ of the linear space spanned by $\left\{e^{-\lambda_{j, k} s}\right\}_{j=1}^{\infty} \underset{k=-\infty}{\infty}$, where

$$
C_{0}[0, \infty]=\left\{F \in C[0, \infty]: \lim _{s \rightarrow \infty} F(s)=0\right\} .
$$

To prove that $X=C_{0}[0, \infty]$, an equivalent formulation of the theorem, it suffices to show that for any $F \in C_{0}[0, \infty]$, there is a $G \in X$ such that

$$
\|F-G\|_{\infty} \leqq \frac{2}{3}\|F\|_{\infty} \text {. }
$$

This is because successive approximations give a sequence in $X$ converging to $F$.

Let $F$ be an element of $C_{0}[0, \infty]$. Since $\lim _{s \rightarrow \infty} F(s)=0$, we may choose $J$ so large that

$$
|F(s)| \leqq{ }_{3}^{1}\|F\|_{\infty} \quad \text { for all } s>l_{J}-1 .
$$

For convenience, denote $l_{J}$ by $l$, and $\alpha_{J}$ by $\alpha$. Define $H(s)$ on $[0, l]$ by

$$
\begin{aligned}
H(s) & =e^{\alpha s} F(s), \quad 0 \leqq s<l-1, \\
& =e^{\alpha s} F(s)(l-s)+F(0)(s-l+1), \quad l-1 \leqq s \leqq l .
\end{aligned}
$$

$H$ is continuous, and $H(0)=H(l)$. Define $L$ to be the periodic extension of $H$. Then $L$ can be uniformly approximated by linear combinations of 
$\exp (i 2 \pi k s / l), \quad k=0, \pm 1, \pm 2, \cdots$. Therefore $G(s)=\frac{1}{3} e^{-\alpha s} L(s)$ can be uniformly approximated by linear combinations of $\exp \left(-\lambda_{J, k} s\right), k=$ $0, \pm 1, \pm 2, \cdots$, and hence belongs to $X$. Clearly

$$
G(s)=\frac{1}{3} F(s) \text { for } s \in[0, l-1] .
$$

For $s \in[l-1, l]$,

$$
\begin{aligned}
|G(s)| & \leqq \frac{1}{3}(|F(s)|(l-s)+|F(0)|(s-l+1)) \\
& \leqq \frac{1}{3}\left(\|F\|_{\infty}(l-s)+\|F\|_{\infty}(s-l+1)\right)=\frac{1}{3}\|F\|_{\infty} .
\end{aligned}
$$

Since $|G(s)| \leqq|G(s-l)|$ for $s>l$, it follows that

$$
\|G\|_{\infty}=\frac{1}{3}\|F\|_{\infty} .
$$

From (30) we have

$$
|F(s)-G(s)| \leqq{ }_{3}^{2}\|F\|_{\infty}, \quad s \in[0, l-1] .
$$

Combining (32) and (28) gives

$$
|F(s)-G(s)| \leqq|F(s)|+|G(s)| \leqq \frac{2}{3}\|F\|_{\infty}, \quad s>l-1 .
$$

Combining (33) and (34) we see that $\|F-G\|_{\infty} \leqq \frac{2}{3}\|F\|_{\infty}$, as asserted in (27).

\section{BIBLIOGRAPHY}

1. Ch. H. Müntz, Über den Approximationssatz von Weierstrass, H. A. Schwarz Festschrift, Berlin, 1914, pp. 303-312.

2. L. Schwartz, Étude des sommes d'exponentielles, 2ième éd., Publ. Inst. Math. Univ. Strasbourg, 5, Actualités Sci. Indust., no. 959, Hermann, Paris, 1959. MR 21 \#5116.

3. O. Szäsz, Über die Approximation stetiger Funktionen durch lineare Aggregate von Potenzen, Math. Ann. 77 (1916), 482-496.

Courant Institute of Mathematical Sciences, New York University, New YORK, NEW YORK 10012 